\title{
Conditioning optimal structure electronics for piezo-devices
}

\author{
Andrés García, Andrés Roteta Lannes and Ezequiel Arnaude \\ GIMAP (Grupo de Investigación en Multifísica Aplicada) \\ 11 de Abril 461, Bahía Blanca, Buenos Aires \\ Argentina
}

\begin{abstract}
In this paper, an optimal conditioning electronics configuration is derived for piezoelectric energy harvesting. Unlike conventional studies where optimal energy (power) is extracted from a given piezo-conditioning circuit, this research focuses on the determination of the supremum: the optimal configuration extracting the biggest possible amount of energy (power) to the load.
\end{abstract}

Along with the electronic design guided by the optimal solution (Pontryaing's principle), LTSpice simulations are compared side by side with a pre-specified configuration using an LDO published previously by the authors.

Keywords- Piezo-device, LDO, Optimal control, State-space variables.

\section{INTRODUCTION}

$\mathrm{O}$ VER the last two decades, piezoelectric devices and harvesting energy have been developing constantly. There are numerous researches about the different uses of these and even regarding others investigations to summarize the state-of-the-art (see for instance [1, 2, 3]).

As mentioned in [3], piezoelectric devices has several applications, it could be from micro/nanoscale sensors, automotive applications and also medical purpose. Another field of potential application is to use piezoelectrics as an energy source for wireless instruments that monitor volcano activities 4 .

In the present research, unlike the available literature, an optimal control point of view is presented so to identify the optimal conditioning configuration circuit. In this regard, instead of defining a controller once a predefined conditioning circuit was presented, the optimal control will account for the complete optimal solution: the conditioning circuit is the outcome of the optimal process.

Considering two different ways to apply a piezoelectric in a circuit: a previously published conditioning circuit in [6] along with the optimal solution in this paper, LT-Spice simulations will provide a solid confirmation that bang-bang controllers (optimal) collects the biggest amount of possible output power.

This paper is organized as follows: Section III. presents the electromechanical model behind the piezo- electric harvesting, Section III. proves that a bang-bang control is the best solution to harvest the biggest output's power amount, Section IV. offers a optimal electronic implementation resorting to a virtual mass concept using a Darlington array, Section V. provides simulations in LT-Spice to compare the circuit published in 6] along with the optimal result in this paper, whereas Section VI. presents some conclusions and future work.

\section{Model Formulation}

A piezo-device is in fact and electromechanical system (see for instance [10]), then both electrical and mechanical models must be written and coupled (see [9] and Figure 1):

$$
\left\{\begin{array}{l}
m \cdot \ddot{x}(t)=-\frac{d U(x)}{d x}-\gamma \cdot \dot{x}(t)-C(x, V)+\zeta_{z} \\
\dot{V}(t)=F(\dot{x}(t), V(t))
\end{array}\right.
$$

where $U(x)$ is the stored energy inside the piezodevice, $\zeta_{z}$ the vibration force modelled as a general random signal, $C(x, V)$ the reaction force due to the transducer, $x(t)$ the position of the piezo-device mass respect to an inertial frame and $\gamma$ a constant.

Moreover, according to 9 , the model can be further simplified to:

$$
\left\{\begin{array}{l}
m \cdot \ddot{x}(t)=-K \cdot x-\gamma \cdot \dot{x}(t)-K_{v} \cdot V(t)+\zeta_{z} \\
\dot{V}(t)=K_{c} \cdot \dot{x}(t)+\phi(V(t))
\end{array}\right.
$$

where $\phi(V(t))$ is a term depending on the electrical load attached to the piezo-device leads, $\left\{K_{v}, K_{c}\right\}$ are constants, and $U(x)=\frac{1}{2} \cdot x^{2}$.

This model, can be further analysed using a current source equivalent circuit as shown in Figure 2 where $C$ is a constant representing the internal capacitance. Then:

$$
\phi(V(t))=\frac{1}{C} \cdot I_{\text {Load }}=\frac{1}{C} \cdot I(t)
$$

Finally the complete model in state-space arises (from (2) and (3p):

$$
\dot{X}(t)=\left\{\begin{array}{l}
x_{2}(t) \\
-\frac{K}{m} \cdot x_{1}-\frac{\gamma}{m} \cdot x_{2}-\frac{K_{v}}{m} \cdot x_{5}+\frac{\zeta_{z}}{m} \\
u(t) \\
K_{c} \cdot x_{2}+\frac{1}{C} \cdot x_{3}
\end{array}\right.
$$




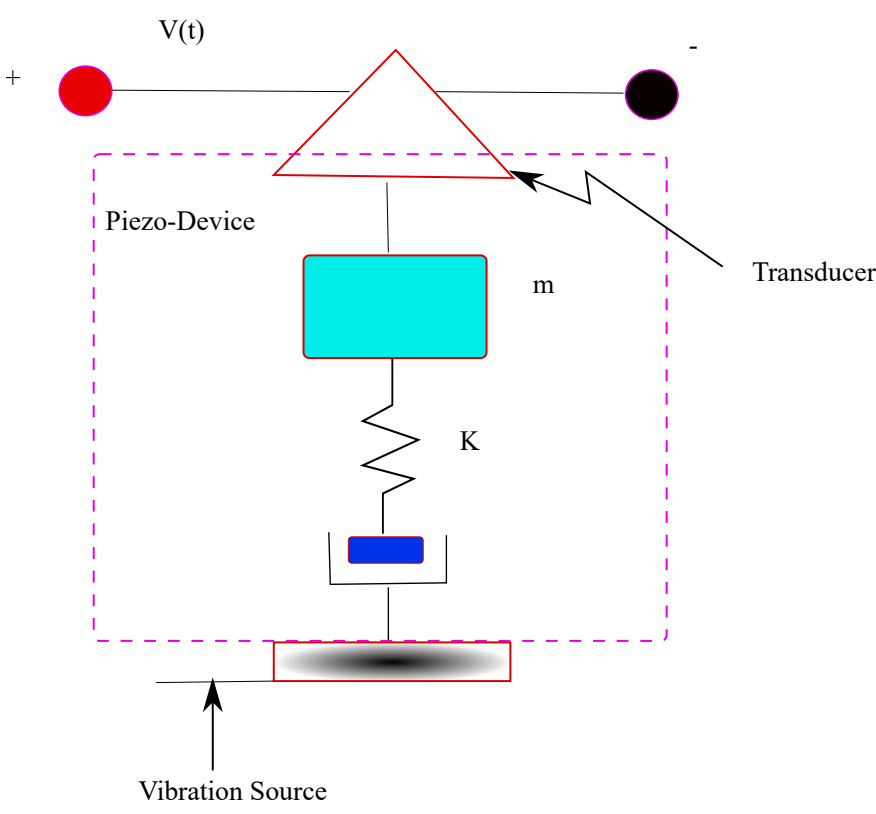

Fig. 1: Piezo-device electro-mechanical model

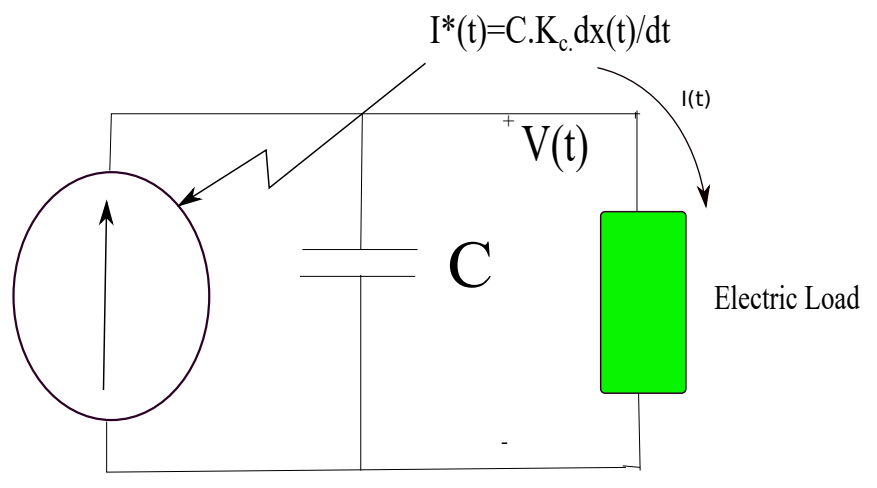

Fig. 2: Piezo-device electro-mechanical model where $X=\left[x_{1}, x_{2}, x_{3}, x_{4}, x_{5}\right]^{\prime}=[x, \dot{x}, I, V]^{\prime}$ and $\dot{I}(t)=u(t)=f(I(t), V, x(t), \dot{x}(t))$ encompass any general non-linear load that must be determined in order to extract the maximum energy form the piezo-device.

\section{Optimal Control}

In order to harvest the maximum possible output power, the following cost function can be defined (from (4) along with a regulated output voltage using an LDO (see for instance [5]):

$$
\begin{aligned}
& \max _{u \in[-U, U]} V(t) \cdot I(t)=\max _{u \in[-U, U]} \quad x_{4}(t) \cdot x_{3}(t) \\
& \text { such that: } \\
& \dot{X}(t)=F(X(t))+B \cdot u(t)+B^{*} \cdot \zeta_{z}(t) \\
& x_{4} \cdot x_{3}=V(t) \cdot I(t)=\eta \cdot \frac{V_{0}^{2}}{R_{L}}
\end{aligned}
$$

Where $F(X(t))=\left[x_{2},\right]^{\prime}, B=[0,0,0,1]^{\prime}, B^{*}=$ $\left[0, \frac{1}{m}, 0,0\right], \eta$ is the LDO's efficiency, $V_{0}$ the regulated output voltage and $R_{L}$ the constant resistance load.

In this context, Pontryagin's principle with an active constraint leads (see [7], pp. 48):

$$
\begin{array}{r}
\max _{u \in[-U, U]} \lambda(t)^{\prime} \cdot B \cdot u(t) \\
\lambda(t)=\left[0,0, x_{4}, x_{3}\right]^{\prime} \\
x_{4} \cdot x_{3}=\eta \cdot \frac{V_{0}^{2}}{R_{L}}
\end{array}
$$

Where the input-output modelling of an LDO (see for instance [5]) is depicted in Figure 3 and can be written as follows:

$$
V_{0}=\left\{\begin{array}{l}
0, \quad V(t) \leq V^{*} \Leftrightarrow I(t)=0 \\
3.3 V, \quad V(t)>V^{*} \Leftrightarrow V(t) \cdot I(t)=\frac{V_{0}^{2}}{R_{L}}
\end{array}\right.
$$

Finally:

$$
\dot{I}(t)=u(t)=\left\{\begin{array}{l}
\frac{\left[1-\operatorname{sign}\left(V-V^{*}\right]\right.}{2} \cdot U, \quad V(t)>V^{*} \\
0, \quad V(t) \leq V^{*}
\end{array}\right.
$$

\section{Electronic REALizATion}

The closed-form solution in (5) needs to be implemented in hardware. To this aim, a simple virtual ground can be constructed using a Darlington with a high input impedance working in the linear region, along with a square wave excitation, as depicted in the red squared section of Figure 4 .

$$
V_{1}(t)=R_{1} \cdot I_{b}+\frac{1}{C_{b e}} \cdot \int_{0}^{t} I_{b}(\sigma) \cdot d \sigma
$$

Where the Base-Emitter capacitor $C_{b e} \rightarrow 0$ at low frequencies was invoked. Moreover, because of the virtual ground effect, the base-emitter voltage can be also considered as constant (see for instance [8]): 


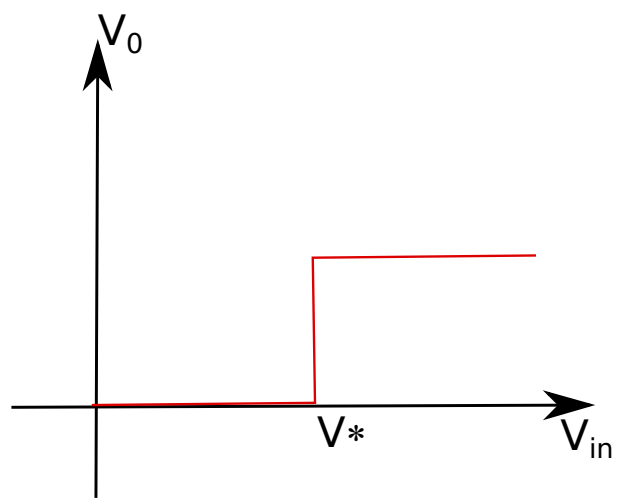

Fig. 3: Typical LDO's modelling

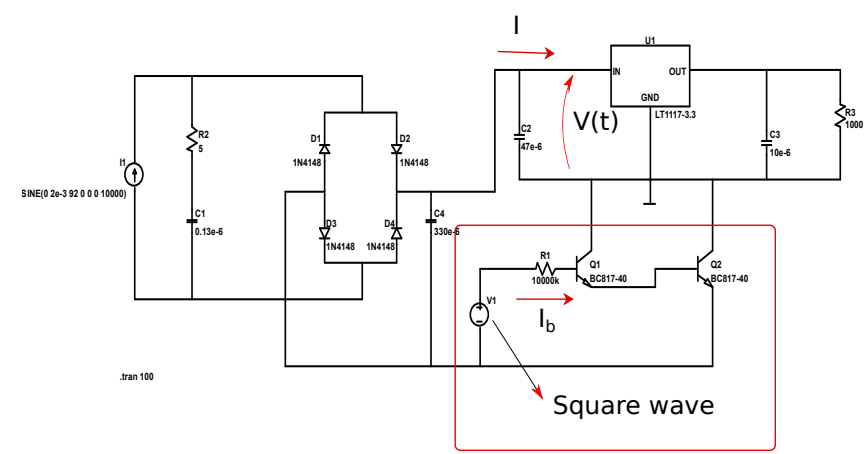

Fig. 4: Final circuit: analysis of virtual's ground effect.

$$
\begin{array}{r}
0 \sim I_{b}+\frac{1}{R_{1} \cdot C_{b e}} \cdot \int_{0}^{t} I_{b}(\sigma) \cdot d \sigma \quad\left(R_{1} \rightarrow \infty\right) \\
\frac{V_{1}-V_{b e}}{R_{1}} \sim I_{b} \\
I(t)=\beta \cdot I_{b}(t)
\end{array}
$$

Where $\beta$ is the classical current amplification in the liner's BJT region. In other words:

$$
\begin{aligned}
\frac{d I_{b}(t)}{d t} \sim \frac{1}{R_{1} \cdot C_{b e}} \cdot I_{b}(t) \quad\left(R_{1}\right. & \rightarrow \infty) \\
\frac{V_{1}-V_{b e}}{R_{1}} & \sim I_{b}
\end{aligned}
$$

Where $R_{1} \cdot C_{b e} \rightarrow$ constant. Then:

$$
\frac{d I(t)}{d t}=\left\{\begin{array}{l}
\sim \frac{\beta}{R_{1}^{2} \cdot C_{b e}} \cdot\left(V_{1}-V_{b e}\right) \quad\left(R_{1} \rightarrow \infty\right), \quad V(t)>V^{*} \\
0, \quad V(t) \leq V^{*}
\end{array}\right.
$$

The final circuit implementation taking into account (6), can be observed in Figure 4 .

\section{Simulation Results}

LT-Spice simulations can be carried out using a sinusoidal current source $I_{1}=2 \mathrm{~mA}$ to simulate the piezodevice excitation and electromechancial conversion at

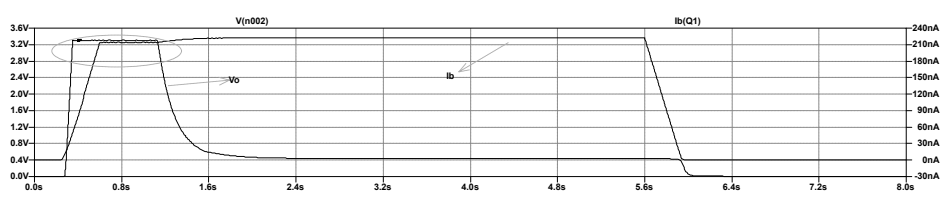

Fig. 5: $V_{0}$ and $I_{b}$ plots using the opitmal circuit.

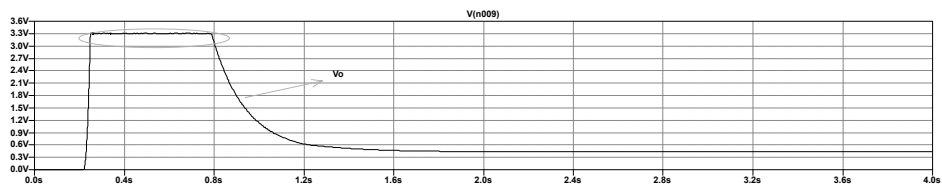

Fig. 6: $V_{0}$ plot using the circuit implemented in [?].

$92 \mathrm{~Hz}$ along with a square wave (optimal input bangbang) of period $1 / 35$ secs. The results are shown in Figures 5 and 6 .

It is remarkable that the time interval where the output voltage $V_{0}$ is active, increases from 0.5365 secs in the circuit at [6] (shown in Figure7), whereas is actually 0.72 secs for the optimal circuit.

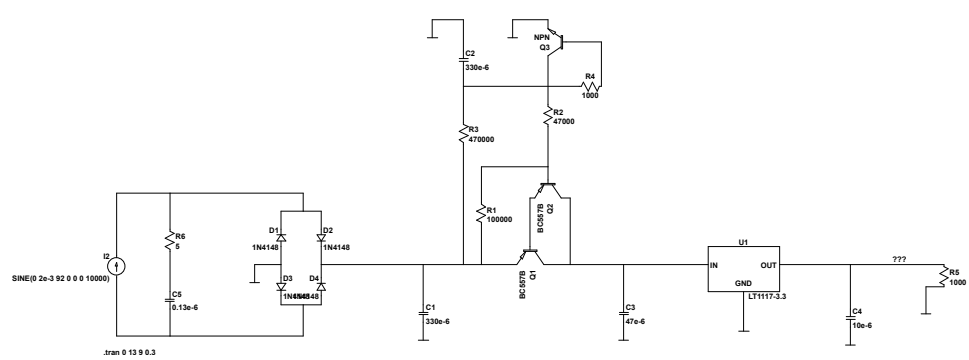

Fig. 7: Previous published circuit using PNP transistors.

\section{COnClusions}

In this paper, a complete electromechanical model of a piezoelectric device plus conditioning circuit was presented and analysed. Unlike conventional studies were a predefined conditioning circuit is decided and then optimal parameters/control is applied (if no optimal control is considered at all), in this paper, the complete conditioning circuit is provided by the Pontryagin's optimal control solution.

Realizing that singular optimal cost functions provides a bang-bang control, a square wave is the best possible control waveform to switch the piezo-device between connection and disconnection to the load (via and LDO), producing an optimal output harvest power.

The simplicity of the optimal solution obtained along with the experience collected using the previous research in 6] opens the way to further studies using bang-bang (square wave) control voltages to harvest piezoelectric energy.

Future work will encompass a further improvement using bang-bang controllers, but at the same time to explore the limits of harvest power/energy using a single piezo-device. 


\section{ACKNOWLEDGMENT}

The authors would like to acknowledge Universidad Tecnológica Nacional under the PID project TC 5122 .

\section{REFERENCES}

[1] Safaei M, Sodano H and Anton S R. A review of energy harvesting using piezoelectric materials: stateof-the-art a decade later (2008-2018). Smart Materials and Structures. 28. 2019.

[2] Toprak A and Tigli O 2014 Piezoelectric energy harvesting: State-of-the-art and challenges.

[3] Chang Y, Zuo J, Zhang H and Duan X.State-of-theart and recent developments in micro/nanoscale pressure sensors for smart wereable devices and health monitoring systems. 2019

[4] D. R. Santoso, S. Maryanto and A.Y. Ponco Wardoyo. Development of a simple and low-cost instrumentation system for real time volcano monitoring. International Journal of Advances in Engineering and Technology, 2(1), 532-542. 2012

[5] Understanding the Terms and Definitions of LDO VoltageRegulator.Application ReportSLVA079 - October 1999. Texas Instruments.

[6] G. Monte, D. Marasco, A. García and E. Perotti.IECON 2018 - 44th Annual Conference of the IEEE Industrial Electronics Society. On-Chip Spectral Analysis with Low Power and Optimal Control for Energy Harvesting Using Piezoelectric Devices. 2018. pp. 3858-3863.

[7] H. P. Geering, Optimal Control With Engineering Applications. Springer, Berlin. 2007.

[8] J. Millman and C. Halkias. Electronic devices and circuits. McGraw-Hill. 1967.

[9] L. Gammaitoni, H. Vocca, I. Neri, F. Travasso and Francesco Orfei. Vibration Energy Harvesting: Linear and Nonlinear Oscillator Approaches. Book chapter Sustainable Energy Harvesting Technologies Past, Present and Future. 2011.

[10] A. Erturk and D. J. Inman. Piezoelectric Energy Harvesting . Wiley; 1st edition. 2011 